\title{
A Matérn model of the spatial covariance structure of point rain rates
}

\author{
Ying Sun • Kenneth P. Bowman . \\ Marc G. Genton • Ali Tokay
}

Received: date / Accepted: date

\begin{abstract}
It is challenging to model a precipitation field due to its intermittent and highly scale-dependent nature. Many models of point rain rates or areal rainfall observations have been proposed and studied for different time scales. Among them, the spectral model based on a stochastic dynamical equation for the instantaneous point rain rate field is attractive, since it naturally leads to a consistent space-time model. In this paper, we note that the spatial covariance structure of the spectral model is equivalent to the well-known Matérn covariance model. Using high-quality rain gauge data, we estimate the parameters of the Matérn model for different time scales and demonstrate that the Matérn model is superior to an exponential model, particularly at short time scales.
\end{abstract}

Keywords Covariance model · Exponential covariance · Matérn covariance · Point rain rates $\cdot$ Spectral model $\cdot$ Time scales

\section{Introduction}

Because of its intermittent nature, high variability, and small length and time scales, precipitation poses significant challenges for both observations and

Y. Sun

Department of Statistics, Ohio State University, Columbus, OH 43210, USA

E-mail: sunwards@stat.osu.edu

K. P. Bowman

Department of Atmospheric Sciences, Texas A\&M University, College Station, TX 77843, USA

M. G. Genton

CEMSE Division, King Abdullah University of Science and Technology, Thuwal 23955-6900, Saudi Arabia

A. Tokay

Joint Center for Earth Systems Technology, University of Maryland Baltimore County, Baltimore, MD 21228, and NASA Goddard Space Flight Center, Greenbelt, MD 20771, USA 
modeling. In addition, rainfall statistics are strongly scale dependent. For example, spatial correlation lengths for monthly rain rates are much larger than for hourly or daily rain rates. Similarly, autocorrelation time scales for areaaveraged rain rates are larger for larger areas. For many precipitation-related problems, such as estimating area-averaged precipitation from a set of rain gauges or validating satellite precipitation observations with surface observations, it is useful to have a statistical model with the characteristics of the precipitation field. Much progress has been made for precipitation modeling; [24] reviewed the development of Poisson-cluster processes, [6] investigated how to calibrate radar measurements via rain gauge data, [23] constructed separate models for rainfall occurrences and the positive rainfall amounts, and [7] developed an adaptive spatial model for precipitation data from multiple satellites.

Many types of inference and estimation problems can be addressed if the covariance structure of the rain field is known [5]. In this study we analyze the spatial covariance of high-quality data from a rain gauge network for different averaging times and compare it with a semi-analytical stochastic model of rain developed by [17], [21], and [4]. Related problems have been addressed by [18], who investigated the space-time covariance structure of propagating precipitation features, and [16], who developed an empirical model of space and time scaling properties of the occurrence of rain. [22] recently discussed a similar problem for the surface temperature field. The distribution of non-zero rain rates is non-Gaussian with a heavy tail at high rain rates. A number of different distributions have been used to represent the conditional rain rate (the rain rate when raining), such as the lognormal or gamma distributions [8] and log-skew-elliptical distributions [19]. The rain model and the resulting form of the space-time covariance function are described in more detail in Section 3.

Developing and calibrating such a statistical model requires consistent, accurate data, particularly when estimating the second moments, e.g., covariances, or even higher moments. In this study we take advantage of high-quality precipitation data from a network of research rain gauges in Virginia, Maryland, and North Carolina that was deployed as part of the NASA Tropical Rainfall Measuring Mission (TRMM) ground validation effort [25]. We use the spatial covariance structure of the gauge data to estimate the parameters of the statistical model of [4] for the purely spatial case, and show that it falls into the family of covariance models described by [20].

\section{Data}

The deployment of the rain gauge network is described in detail in [25]. For quality control and reliability, each site in the network has two or three research-quality 8-inch tipping-bucket rain gauges manufactured by Met One Inc. These gauges are colocated with at least one rain gauge from an operational rainfall monitoring network. From the 20 sites in the network, we select 
12 that have essentially complete data for the three-year period from 2004-0519 to 2007-05-17. The map in Figure 1 shows the locations of the 12 gauges

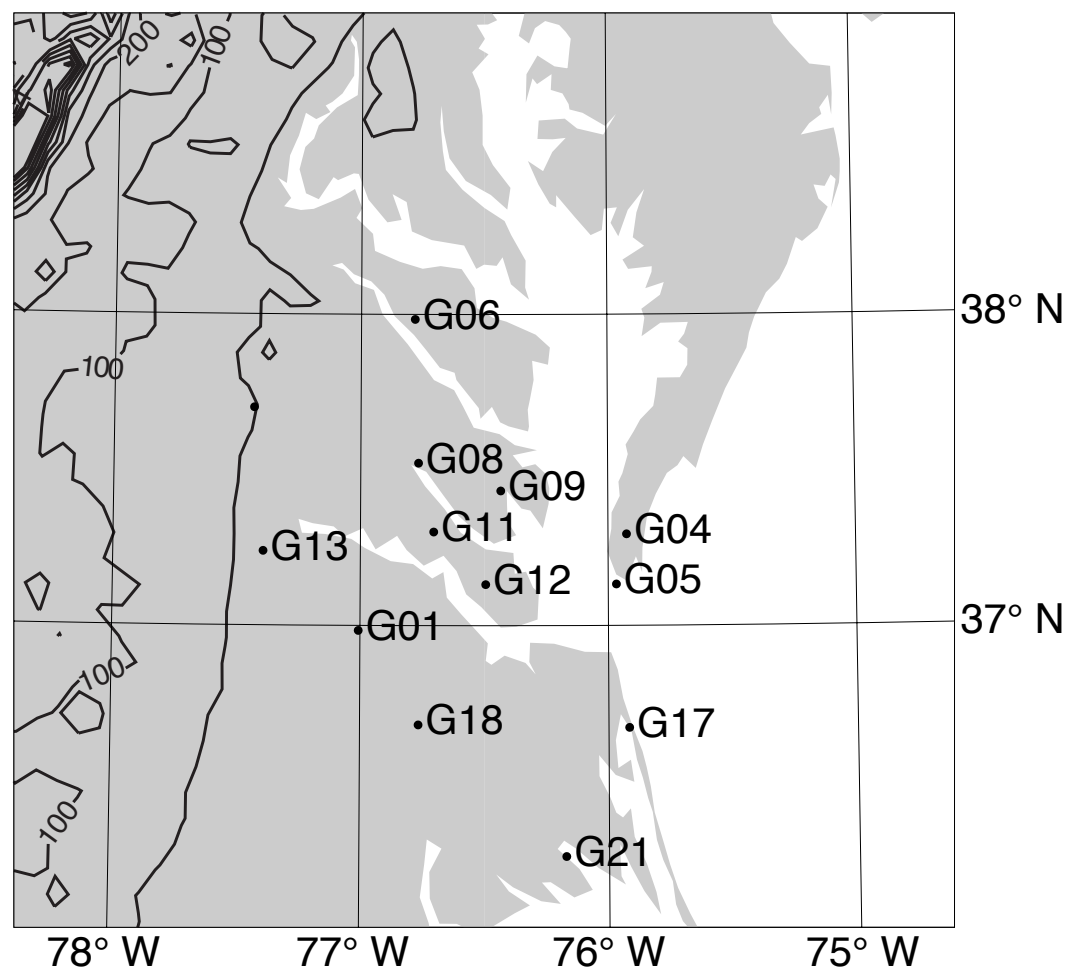

Fig. 1 Locations of the twelve rain gauges used in this study. The solid black lines are contours of surface elevation in meters. The contour interval is $50 \mathrm{~m}$.

used here. The gauges record the time of each bucket tip; one tip is equal to $0.254 \mathrm{~mm}$ (0.01 inches) of rain. Bucket tips are converted to rain rates by counting the number of tips within specified intervals. The longitudes and latitudes of the gauge sites and the mean annual rain rate at each gauge for the period of study are given in Table 1, which shows that the long-term mean rate is relatively constant across the network.

\section{Rain rate covariance model}

3.1 Spectral covariance model

[4] developed a spectral stochastic model for rain rates that is a simplification of the rainfall model in [3]. The analytical properties of a similar model for 
Table 1 Gauge locations and annual mean rain rate at each gauge for the three-year period of study.

\begin{tabular}{cccc}
\hline Gauge & Longitude & Latitude & $\begin{array}{c}\text { Mean } \\
(\mathrm{mm})\end{array}$ \\
\hline G01 & -77.00 & 36.98 & 1309 \\
G04 & -75.92 & 37.29 & 966 \\
G05 & -75.96 & 37.13 & 1195 \\
G06 & -76.78 & 37.98 & 1334 \\
G08 & -76.76 & 37.52 & 1141 \\
G09 & -76.43 & 37.43 & 1318 \\
G11 & -76.70 & 37.30 & 1258 \\
G12 & -76.49 & 37.13 & 1287 \\
G13 & -77.39 & 37.23 & 1267 \\
G17 & -75.91 & 36.67 & 1358 \\
G18 & -76.76 & 36.68 & 1308 \\
G21 & -76.17 & 36.25 & 1305 \\
\hline
\end{tabular}

temperature are discussed in more detail in [22]. The surface rain rate $R(\mathbf{x}, t)$ at location $\mathbf{x}$ and time $t$ is written as a sum of spatial Fourier modes with amplitudes $a(\mathbf{k}, t)$ of the rain field fluctuation defined as the deviation from the mean, where $\mathbf{k}$ is the spatial frequency. If we assume isotropy, then $k=|\mathbf{k}|$, and the amplitude of each mode is given by a first-order differential equation of the form

$$
\frac{d a(k, t)}{d t}=-\frac{1}{\tau_{k}} a(k, t)+f_{k}(t),
$$

where $f_{k}(t)$ is a white-noise forcing. The damping time scale for each mode $\tau_{k}$ is taken to have the form

$$
\tau_{k}=\frac{\tau_{0}}{\left(1+k^{2} \beta_{0}^{2}\right)^{1+\eta}},
$$

where $\tau_{0}$ is the correlation time scale of the area-averaged field $(k=0)$, and $\beta_{0}$ is a characteristic length scale. The exponent $\eta$ affects the scale dependence of the damping timescale $\tau_{k}$, which provides additional control over the shape of the covariance function.

From (1), [4] found that the power spectrum of the stochastic rain field $R$ can be shown to be

$$
\tilde{c}(k, \omega)=\frac{F_{0} \tau_{0}^{2}}{\tau_{0}^{2} \omega^{2}+\left(1+k^{2} \beta_{0}^{2}\right)^{2+2 \eta}},
$$

where $\omega$ is the temporal frequency and $F_{0}$ is the magnitude of the white-noise forcing, which determines the total variance of the rain field. The space-time covariance of the point rain rate at distance $r=|\mathbf{r}|$ and time lag $s$ implied by this model is given by the inverse transform of the power spectrum

$$
c(r, s)=(2 \pi)^{-3 / 2} \int \mathrm{d} \omega \int \mathrm{dk} \mathrm{e}^{(\mathrm{ikr}-\omega \mathrm{s})} \tilde{\mathrm{c}}(\mathrm{k}, \omega) .
$$


This is a stationary spatio-temporal covariance model for instantaneous point rain rates with four parameters: $F_{0}, \tau_{0}, \beta_{0}$ and $\eta$. The covariance of the timeaveraged or the area-averaged rain rates can be expressed as suitable integrals over the spectrum for a given set of parameters that are fixed across different time or spatial scales.

For point rain rates, integration of (4) over $\omega$ gives

$$
c(r, s)=(2 \pi)^{-1} \int d k e^{i k r} c(k, s)
$$

where

$$
c(k, s)=\sqrt{\frac{\pi}{2}} F_{0} \tau_{k} e^{-|s| / \tau_{k}} .
$$

[4] noticed that [1] gives the result

$$
c(r, 0)=\gamma_{0} C_{\eta}\left(\frac{r}{\beta_{0}}\right),
$$

where $C_{\eta}(z)=\left(\frac{z}{2}\right)^{\eta} K_{\eta}(z)$, with $K_{\eta}(z)$ denoting the modified Bessel function of order $\eta$, and $\gamma_{0}$ is related to $F_{0}$ by

$$
F_{0}=\sqrt{\frac{2}{\pi}} \Gamma(1+\eta)\left(\frac{\beta_{0}^{2}}{\tau_{0}}\right) \gamma_{0} .
$$

\subsection{Matérn covariance model}

Among many available covariance models, the Matérn family [20] has gained widespread interest in recent years. [14] introduced the Matérn form of spatial correlations into statistics as a flexible parametric class with one parameter determining the smoothness of the underlying spatial random field. The varied history of this family of models can be found in [12]. The Matérn form also naturally arises as the correlation for temperature fields described by simple energy balance climate models [22]. The Matérn class of covariance functions is defined as

$$
c(r)=\frac{2 \sigma^{2}}{\Gamma(\nu)}\left(\frac{r}{2 L_{0}}\right)^{\nu} K_{\nu}\left(\frac{r}{L_{0}}\right),
$$

where $\nu>0$ depends upon the smoothness of the random field, with larger values of $\nu$ corresponding to smoother fields; and $L_{0}>0$ is a spatial range parameter that measures how quickly the correlation of the random field decays with distance, with larger $L_{0}$ corresponding to a slower decay (keeping $\nu$ fixed). When $\nu=1 / 2$, the Matérn covariance function reduces to the exponential covariance model and describes a rough field. The value $\nu=\infty$ corresponds to a Gaussian covariance model which describes a very smooth field, in fact a field which is infinitely differentiable.

The spatial covariance function of the spectral model in (7) is a Matérn form, with $\eta=\nu, \gamma_{0}=2 \sigma^{2} / \Gamma(\nu)$ and $\beta_{0}=L_{0}$. Thus, (1) in the spatial domain is equivalent to the Matérn class of covariance models defined in (7). 


\section{Methods}

We analyze the spatial covariance structure of rainfall over the gauge network by first computing average rainfall rates at each gauge at time resolutions varying from 5 minutes to 3 weeks. The covariances between each pair of gauges are then computed for each time resolution. Specifically, for each time resolution, we use the observed rain rates within the three-year period to compute the sample correlation between any two sites from the 12 selected gauges. The resulting $\left(\begin{array}{c}12 \\ 2\end{array}\right)=66$ correlation estimates can be plotted as a function of distance between the two corresponding gauges. The minimum and maximum distances between the gauges in the network are 18 and $200 \mathrm{~km}$, respectively. To describe and model the spatial correlation patterns, we fit the general Matérn and the exponential (special case of $\nu=1 / 2$ ) spatial correlation models to the correlation estimates. Parameters are estimated by ordinary least squares (OLS). The general Matérn model fits two parameters, $L_{0}$ and $\nu$. For the exponential model the only adjustable parameter is $L_{0}$. In principle the correlation should go to 1 as the separation between instruments goes to zero, but instrumental error, for example, would cause correlations to be less than 1 even for instruments at the same location. Both models are fit without accounting for these so-called nugget effects by forcing the correlation to go to 1 at $r=0$ since the high-quality colocated gauges do not suggest measurement errors. We aim to show that the flexible Matérn covariance model is more appropriate than choosing the exponential model with an unrealistic nugget effect.

\section{Results}

Figure 2 shows the correlations of time-averaged rain rates between each pair of rain gauges as a function of station separation for four different time-averaging windows ranging from 10 minutes to 1 day. Results for averaging windows longer than 1 day are very similar to those for 1 day (panel d). The solid lines are the least-squares fits to the data using the Matérn model, while the dashed lines are for the exponential model. [15] plotted similar figures for long-term seasonally-averaged precipitation values, while [13] examined correlations at station separations less than $10 \mathrm{~km}$. As expected, the correlations between gauges are larger for gauges that are closer to one another and for longer averaging windows. The model fits also show that as the aggregation time increases, the spatial correlation becomes stronger for a certain distance. In other words, the spatial range $L_{0}$ is larger for longer averaging times.

The parameter estimates and the resulting mean squared errors (MSEs) from the Matérn and exponential model fittings are given in Table 2. For each averaging time window, the smaller MSE of the Matérn model indicates a better fit compared to the exponential model. Although the exponential model always has a bigger MSE than the Matérn model does, the difference generally decreases as the averaging time window increases. The quality of the 
a) 10 minute

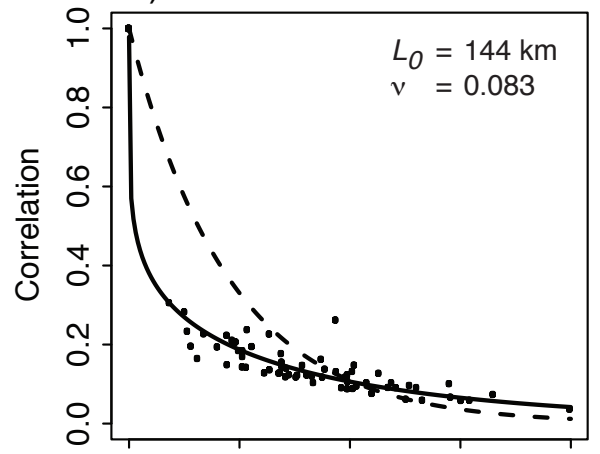

c) 6 hour

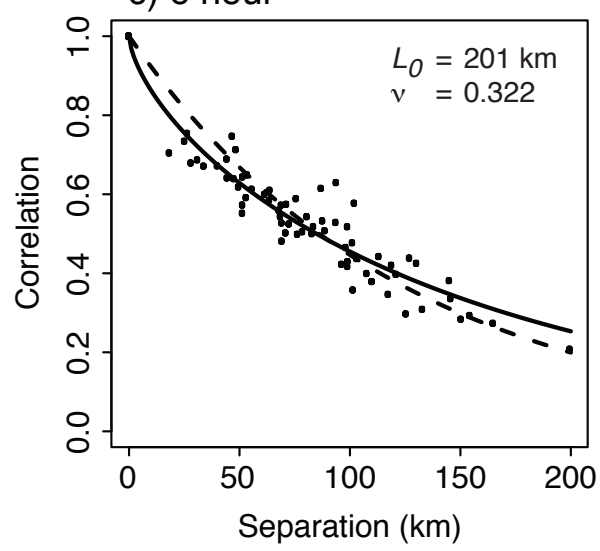

b) 1 hour

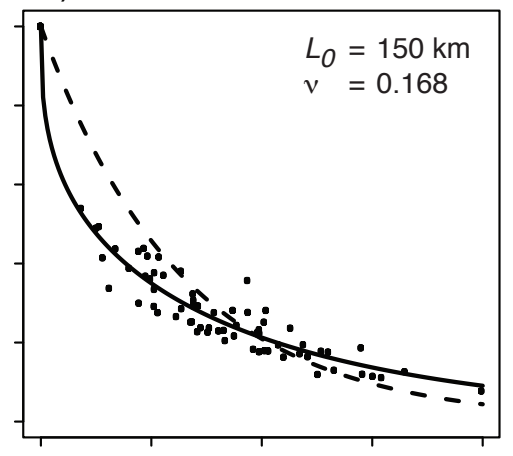

d) 1 day

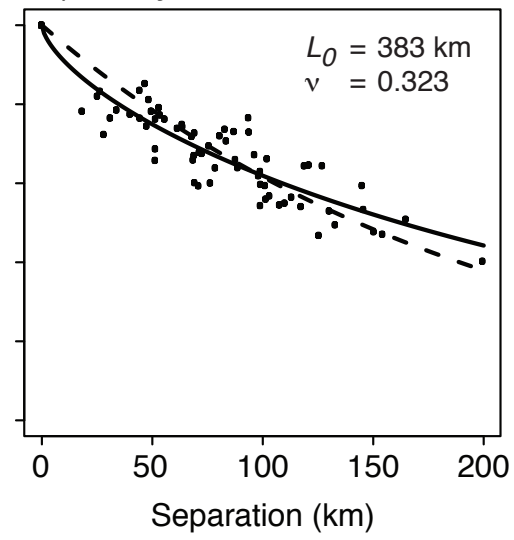

Fig. 2 Correlation as a function of site separation distance for different gauge averaging times. Parameters shown are for the Matérn fits. Solid lines: least-squares fit to Matérn function; dashed lines: least-squares fit to exponential.

fits can be seen graphically in Figure 2, which shows that the Matérn model better captures the shape of the correlation function than the exponential model, particularly for shorter averaging times.

For the Matérn model the MSE becomes bigger for longer time-averaging windows. This is not surprising because for a fixed record length, the number of rain rate samples within the record becomes smaller as the averaging time window gets longer. Moreover, the parameter estimates of the Matern model in Table 2 show that when the time-averaging window increases beyond 6 hours, the length scale becomes larger than the maximum separation between the gauges $(202 \mathrm{~km})$. This can also be seen in the plots in Figure 2. For longer 
Table 2 Parameter estimates and mean squared errors (MSEs).

\begin{tabular}{l|ccc|cc}
\hline \multirow{2}{*}{$\begin{array}{l}\text { Averaging } \\
\text { window }\end{array}$} & \multicolumn{3}{|c|}{ Matérn Model } & \multicolumn{2}{c}{ Exponential Model } \\
\cline { 2 - 6 } & $L_{0}(\mathrm{~km})$ & $\nu$ & MSE $\left(\times 10^{3}\right)$ & $L_{0}(\mathrm{~km})$ & MSE $\left(\times 10^{3}\right)$ \\
\hline 5 minutes & 125 & 0.080 & 0.85 & 42 & 14.04 \\
10 minutes & 144 & 0.083 & 0.91 & 45 & 13.49 \\
15 minutes & 159 & 0.087 & 0.93 & 48 & 12.96 \\
30 minutes & 173 & 0.105 & 1.14 & 54 & 11.06 \\
1 hour & 150 & 0.168 & 1.48 & 64 & 7.15 \\
3 hours & 165 & 0.268 & 2.05 & 91 & 3.87 \\
6 hours & 201 & 0.322 & 2.22 & 124 & 3.04 \\
1 day & 383 & 0.323 & 2.50 & 206 & 3.15 \\
1 week & 396 & 0.320 & 2.50 & 207 & 3.16 \\
3 weeks & 267 & 0.390 & 5.06 & 189 & 5.80 \\
\hline
\end{tabular}

time scales, therefore, the gauge network is probably too small to accurately estimate the natural length scale of variability of the rain field.

Figure 3 shows graphically the parameter estimates of $L_{0}$ and $\nu$ from the Matérn model shown in Table 2 for the given averaging time windows (unit: hour). For time scales out to a day, the spatial range parameter $L_{0}$ increases approximately linearly. The value of $L_{0}$ does not have an increase from 30 minutes to 1 hour or 3 hours because the spatial correlation depends on both $L_{0}$ and $\nu$ in the Matérn model, unlike the exponential model where $L_{0}$ itself controls the spatial range. Indeed, up to 1 week, $L_{0}$ in the exponential model always increases as the averaging time window gets longer. However, the value of $L_{0}$ becomes smaller for the 3 weeks time window, possibly due to the fact that fewer available observations make the estimation less accurate. Therefore, we need a period of time longer than 3 years to estimate the parameters for the averaging time window greater than 1 week. Moreover, the limiting value for very short averaging times appears to be near $100 \mathrm{~km}$, but it is important to keep in mind that the estimates of the parameter $\nu$ indicate that the precipitation field is very non-smooth. The smoothness parameter itself increases from a value less than 0.1 to around 0.3 for longer averaging times. In all cases the estimates of the exponent $\nu$ from fitting the Matérn model are less than the value for the exponential model (0.5). This indicates that rain fields are rougher than a random field that has an exponential spatial covariance. At short time scales the rain field appears to be much rougher than exponential, but this conclusion is limited by the fact that the minimum separation of the rain gauges is $18 \mathrm{~km}$, so it is not possible to directly observe the variability at length scales shorter than that. The smoothness parameter $\nu$ increases as the averaging time increases, indicating a smoother random field. This is not surprising, as time averaging would be expected to reduce the variability of the rain field.

The results in Table 2 can be compared with Table 1 in [5]. They fit a single consistent space-time covariance model of [4], and the parameters of the covariance model are estimated using radar rainfall maps from the several different tropical field experiments. The model in [4] describes the covariance 

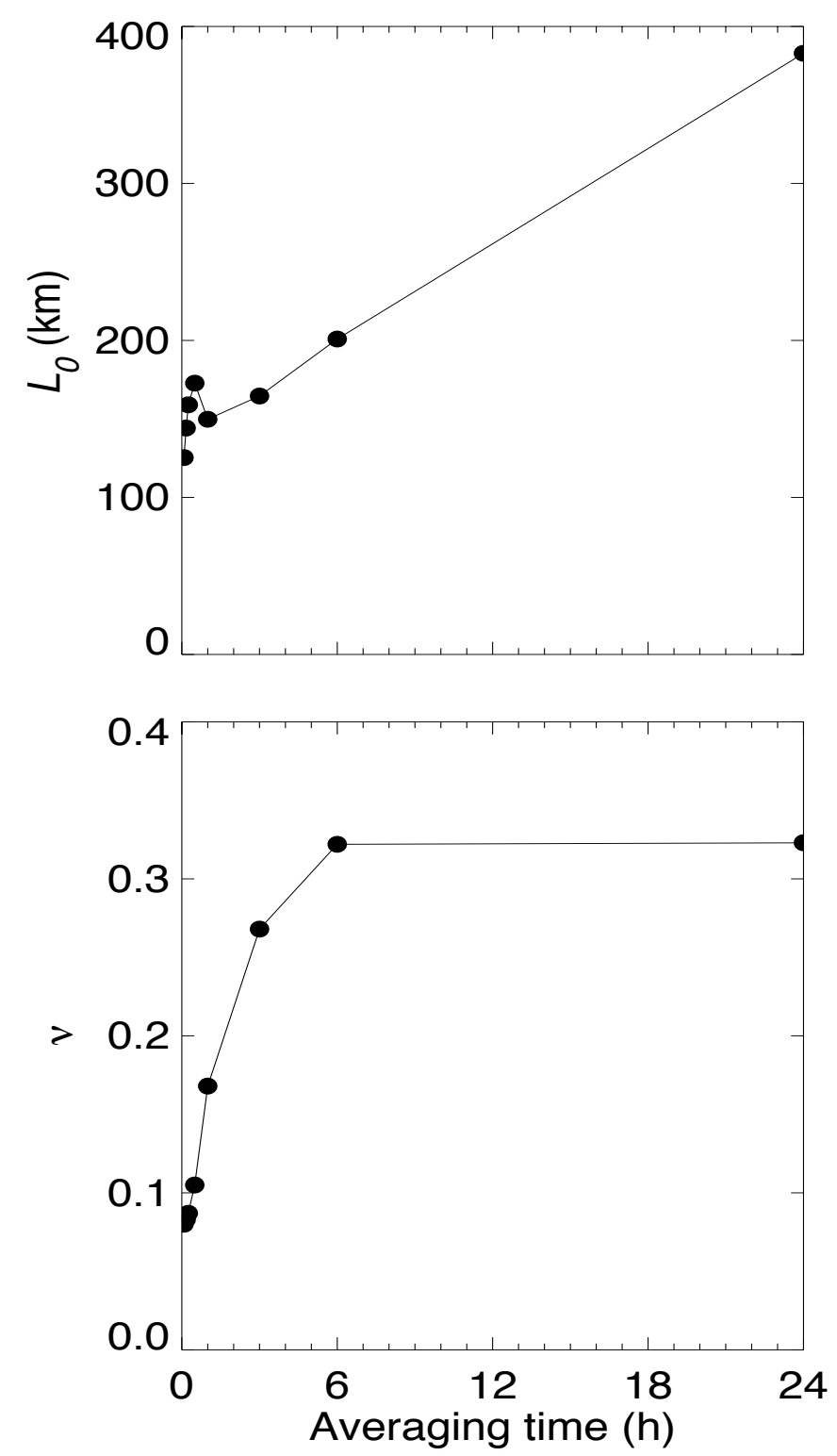

Fig. 3 Top panel: the plot of parameter estimates of $L_{0}$ from the Matérn model (shown in Table 2) as a function of averaging time (unit: hour). Bottom panel: the plot of parameter estimates of $\nu$ from the Matérn model (shown in Table 2) as a function of averaging time (unit: hour).

structure of the instantaneous point rain rates, so that the covariance of the time-averaged rain gauge measurements and the area-averaged radar observa- 
tions can be expressed as suitable integrals. Therefore, its parameters are scale independent. In our analysis, we fit independent spatial covariance models for different time scales and obtain different parameter estimates, which are thus dependent on time scales.

In [5], the spatial and temporal resolution of the radar data from those experiments is typically $\sim 4 \mathrm{~km}$ and $\sim 15$ minutes, respectively. They found values of $\beta_{0}$ from 61 to $104 \mathrm{~km}$ for instantaneous rain rates. In our analysis, when averaging the rain gauge data with windows of 15 minutes or less, the parameter $L_{0}$ in the Matérn model ranges from 125 to $159 \mathrm{~km}$, which corresponds reasonably well to the radar data with a slightly larger spatial range due to the short-time aggregation. The greatest differences between this study and [5] are found for the values of the smoothness parameter. [5] estimated $\eta$ to be in the range $-1 / 2 \leq \eta<0$, arguing that negative values of $\eta$ fit the data best. Although for the space-time spectrum in (3), the part, $\left(1+k^{2} \beta_{0}^{2}\right)^{2+2 \eta}$, in the denominator still makes the spectrum integrable with respect to $k$, the purely spatial model, which is a Matérn form with the spectrum having $\left(1+k^{2} \beta_{0}^{2}\right)^{1+\eta}$ in the denominator, has infinite variance at the origin. In our fitting procedure, however, we only fit a purely spatial covariance model, therefore, we constrain the values of $\nu$ to be positive, which results in a finite variance at the origin and a valid covariance model.

\section{Discussion}

In geostatistics, it is common to treat observations in time as replicates and fit a spatial exponential covariance model to precipitation data. In this paper, we aimed to show that the flexible Matérn covariance model is more appropriate than the exponential model for characterizing the spatial dependence of the precipitation especially on short time scales. Although maximum likelihood estimates have better properties, it is not trivial to apply the maximum likelihood method due to the fact that the distribution of precipitation is usually non-Gaussian with rainfall zeros. Therefore, the least squares method is a reasonable alternative, widely used in the precipitation literature, in the sense that we do not have to assume distributions and temporal replicates are available in our setting. In the least squares method, the MSE plays the same role as the negative likelihood function, where a smaller MSE indicates a better model. We have shown that the spatial component of the spectral covariance model for precipitation developed by [4], [5], is equivalent to the family of covariance models introduced by [20]. We used high-quality, high-frequency rain rate data from a network of research rain gauges [25] to estimate the parameters of the Matérn covariance model for a range of different rain-rate averaging times (accumulation times). The results indicated that for averaging times less than a few weeks, the rain field is rougher than is the case for a random field with an exponential spatial covariance structure. The roughness increases as the averaging time decreases. In contrast to [5], our model is time scale dependent. We estimate the parameters of the Matérn model using 
the rain gauge data with different aggregation to characterize the precipitation field at different time scales in terms of the smoothness and the spatial correlation range.

The datasets described in this paper have many interesting features to explore. We have focused on estimating the spatial correlation of the precipitation using the Matérn covariance model to compare the roughness of the spatial fields on different time scales. Considering the relatively small geographic region in this study, the spatial stationarity of the precipitation process was assumed. If the spatio-temporal dependence is of interest, one may further develop more complicated spatio-temporal models for each given time scales. However, the stationarity assumption in time is unlikely to be realistic as precipitation usually shows seasonal trend. Consequently, a longer period of data will be needed to study the long-term characteristics.

It is also worth noting that in addition to the Matérn class, there are other available covariance families that one can choose from, depending on the properties of the precipitation field. For example, the generalized Cauchy family proposed by [10] is suitable to model the long-range dependence in a process. Whether such a covariance family may arise from a stochastic differential equation similar to (1) is an open problem. Further extensions to multiple variables and multivariate Matérn cross-covariance functions [11,2,9] would be of interest too.

Acknowledgements The research in this article was partially supported by Award No. KUSC1-016-04 made by King Abdullah University of Science and Technology (KAUST) and by the Spanish Ministry of Science and Innovation (Project MTM2011-22664) which is co-funded by FEDER.

\section{References}

1. Abramowitz, M., Stegun, I.A.: Handbook of Mathematical Functions with Formulas, Graphs, and Mathematical Tables. No. 55 in National Bureau of Standards Applied Mathematics Series. Superintendent of Documents, U.S. Government Printing Office, Washington, DC (1964)

2. Apanasovich, T.V., Genton, M.G., Sun, Y.: A valid Matérn class of cross-covariance functions for multivariate random fields with any number of components. Journal of the American Statistical Association 107, 180-193 (2012)

3. Bell, T.L.: A space-time stochastic model of rainfall for satellite remote-sensing studies. Journal of Geophysical Research 92 (1987). DOI 10.1029/JD092iD08p09631

4. Bell, T.L., Kundu, P.K.: A study of the sampling error in satellite rainfall estimates using optimal averaging of data and a stochastic model. Journal of Climate 9, 12511268 (1996)

5. Bell, T.L., Kundu, P.K.: Comparing satellite rainfall estimates with rain gauge data: Optimal strategies suggested by a spectral model. Journal of Geophysical Research 108 4121 (2003). DOI 10.1029/2002JD002641

6. Bruno, F., Cocchi, D., Greco, F., Scardovi, E.: Spatial reconstruction of rainfall fields from rain gauge and radar data. Stochastic Environmental Research and Risk Assessment 28, 1235-1245 (2014)

7. Chakraborty, A., De, S., Bowman, K., Sang, H., Genton, M.G., Mallick, B.: An adaptive spatial model for precipitation data from multiple satellites over large regions. Statistics and Computing (2014). To appear 
8. Essenwanger, O.M.: General Climatology, 1B, Elements of Statistical Analysis, World Survey of Climatology, vol. 1B. Elsevier, Amsterdam (1985)

9. Genton, M.G., Kleiber, W.: Cross-covariance functions for multivariate geostatistics. Statistical Science (2014). To appear

10. Gneiting, T.: Stochastic models that separate fractal dimension and the hurst effect. SIAM Review 46, 269-282 (2004)

11. Gneiting, T., Kleiber, W., Schlather, M.: Matérn cross-covariance functions for multivariate random fields. Journal of the American Statistical Association 105, 1167-1177 (2010)

12. Guttorp, P., Gneiting, T.: Studies in the history of probability and statistics XLIX: On the Matérn correlation family. Biometrika 93, 989-995 (2006). DOI 10.1093/biomet/93.4.989

13. Habib, E., Krajewski, W.F., Ciach, G.J.: Estimation of rainfall interstation correlation. Journal of Hydrometeorology 2, 621-629 (2001)

14. Handcock, M.S., Stein, M.L.: A Bayesian analysis of kriging. Technometrics 35, 403-410 (1993)

15. Krajewski, W.F., Ciach, G.J., McCollum, J.R., Bacotiu, C.: Initial validation of the Global Precipitation Climatology Project monthly rainfall over the United States. Journal of Applied Meteorology 39, 1071-1086 (2000)

16. Kundu, P.K., Siddani, R.K.: Scale dependence of spatiotemporal intermittence of rain. Water Resources Research 47, 318-340 (2011). DOI 10.1029/2010WR010070

17. Laughlin, C.R.: On the effect of temporal sampling on the observation of mean rainfall. In: D. Atlas, O.W. Thiele (eds.) Precipitation Measurement from Space, pp. D59-66. National Aeronautics and Space Administration, Greenbelt, MD (1981)

18. Li, B., Murthi, A., Bowman, K., North, G., Genton, M.G., Sherman, M.: Statistical tests of Taylor's hypothesis: an application to precipitation fields. Journal of Hydrometeorology 10, 254-265 (2009)

19. Marchenko, Y.V., Genton, M.G.: Multivariate log-skew-elliptical distributions with applications to precipitation data. Environmetrics 21, 318-340 (2010)

20. Matérn, B.: Spatial Variation, Lecture Notes in Statistics, vol. 36, second edition edn. Springer-Verlag, Berlin; New York (1986)

21. North, G.R., Nakamoto, S.: Formalism for comparing rain estimation designs. Journal of Atmospheric and Oceanic Technology 6, 985-992 (1989)

22. North, G.R., Wang, J., Genton, M.G.: Correlation models for temperature fields. Journal of Climate 24, 5850-5862 (2011). DOI 10.1175/2011JCLI4199.1

23. Oliveira, V.D.: A simple model for spatial rainfall fields. Stochastic Environmental Research and Risk Assessment 18, 131-140 (2004)

24. Onof, C., Chandler, R.E., Kakou, A., Northrop, P., Wheater, H.S., Isham, V.: Rainfall modelling using poisson-cluster processes: a review of developments. Stochastic Environmental Research and Risk Assessment 14, 384-411 (2000)

25. Tokay, A., Bashor, P.G., McDowell, V.L.: Comparison of rain gauge measurements in the mid-Atlantic region. Journal of Hydrometeorology 11, 553-565 (2010). DOI 10.1175/2009JHM1137.1 